\title{
Type Variables in Patterns
}

\author{
Richard A. Eisenberg \\ Bryn Mawr College \\ Bryn Mawr, PA, USA \\ rae@cs.brynmawr.edu
}

\author{
Joachim Breitner \\ University of Pennsylvania \\ Philadelphia, PA, USA \\ joachim@cis.upenn.edu
}

\author{
Simon Peyton Jones \\ Microsoft Research \\ Cambridge, UK \\ simonpj@microsoft.com
}

\begin{abstract}
For many years, GHC has implemented an extension to Haskell that allows type variables to be bound in type signatures and patterns, and to scope over terms. This extension was never properly specified. We rectify that oversight here. With the formal specification in hand, the otherwiselabyrinthine path toward a design for binding type variables in patterns becomes blindingly clear. We thus extend ScopedTypeVariables to bind type variables explicitly, obviating the Proxy workaround to the dustbin of history.
\end{abstract}

CCS Concepts - Software and its engineering $\rightarrow$ Patterns; Functional languages; Data types and structures;

Keywords Patterns, type variables, polymorphism, Haskell ACM Reference Format:

Richard A. Eisenberg, Joachim Breitner, and Simon Peyton Jones. 2018. Type Variables in Patterns. In Proceedings of the 11th ACM SIGPLAN International Haskell Symposium (Haskell '18), September 27-28, 2018, St. Louis, MO, USA. ACM, New York, NY, USA, 12 pages. https://doi.org/10.1145/3242744.3242753

\section{Introduction}

Haskell allows the programmer to write a type signature for a definition or expression, both as machine-checked documentation, and to resolve ambiguity (Section 2.1). For example,

$$
\begin{gathered}
\text { prefix }:: a \rightarrow[[a]] \rightarrow[[a]] \\
\text { prefix } x \text { yss }=\text { map xcons yss } \\
\text { where xcons ys }=x: y s
\end{gathered}
$$

Sadly, it is not always possible to write such a type signature. For example, to give a signature for xcons we might try:

$$
\begin{gathered}
\text { prefix }:: a \rightarrow[[a]] \rightarrow[[a]] \\
\text { prefix } x \text { yss }=\text { map xcons yss } \\
\text { where xcons }::[a] \rightarrow[a] \\
\text { xcons ys }=x: y s
\end{gathered}
$$

Permission to make digital or hard copies of all or part of this work for personal or classroom use is granted without fee provided that copies are not made or distributed for profit or commercial advantage and that copies bear this notice and the full citation on the first page. Copyrights for components of this work owned by others than ACM must be honored. Abstracting with credit is permitted. To copy otherwise, or republish, to post on servers or to redistribute to lists, requires prior specific permission and/or a fee. Request permissions from permissions@acm.org.

Haskell '18, September 27-28, 2018, St. Louis, MO, USA

() 2018 Association for Computing Machinery.

ACM ISBN 978-1-4503-5835-4/18/09 ..\$15.00

https://doi.org/10.1145/3242744.3242753
But Haskell98's scoping rules specify that the $a$ in the signature for xcons is locally quantified, thus: $x c o n s:: \forall a$. $[a] \rightarrow$ $[a]$. That is not what we want! We want the $a$ in the signature for $x$ cons to mean "the universally quantified type variable for prefix", and Haskell98 provides no way to do that.

The inability to supply a type signature for xcons might seem merely inconvenient, but it is just the tip of an iceberg. Haskell uses type inference to infer types, and that is a wonderful thing. However, insisting on complete type inferencethat is, the ability to infer types for any well-typed program with no help from the programmer-places serious limits on the expressiveness of the type system. GHC's version of Haskell has gone far beyond these limits, and fundamentally relies on programmer-supplied annotations to guide type inference. As some examples, see the work of Peyton Jones et al. [2007], Vytiniotis et al. [2011], or Eisenberg et al. [2016].

So the challenge we address is this: it should be possible for the programmer to write an explicit type signature for any sub-term of the program. To do so, some type signatures must refer to a type that is already in the static environment, so we need a way to name such types. The obvious way to address this challenge is by providing language support for lexically scoped type variables. GHC has long supported scoped type variables: the ScopedTypeVariables extension is very popular, and $29 \%$ of Haskell packages on Hackage use it. But it has never been formally specified! Moreover, as we shall see, it is in any case inadequate to the task. In this paper we fix both problems, making the following contributions:

- In the days of Haskell98, scoped type variables were seldom crucial. Through a series of examples we show that, as Haskell's type system has grown more sophisticated, the need for scoped type variables has become acute (Section 2), while GHC's existing support for them has become more visibly inadequate (Section 3).

- To fix these inadequacies, we describe visible type application in patterns, a natural extension to GHC's existing visible type applications from terms to patterns (Section 4).

- We give the first formal specification of scoped type variables for Haskell, formalizing the folklore, and providing a firm foundation for both design and implementation (Section 5).

- As part of this specification, we offer a new and simpler typing judgment for GADT pattern matching (Section 5.3), which treats uniformly the universal and existential variables of a data constructor. 


$$
\begin{aligned}
& \text { show }:: \text { Show } a \Rightarrow a \rightarrow \text { String } \\
& \text { read }:: \text { Read } a \Rightarrow \text { String } \rightarrow a \\
& (+) \quad:[a] \rightarrow[a] \rightarrow[a] \\
& \text { concat }::[[a]] \rightarrow[a]
\end{aligned}
$$

Figure 1. Types of standard functions

\section{Motivation and Background}

\subsection{The Need for Type Annotations}

One of the magical properties of ML-family languages, including Haskell, is that type inference allows us to write many programs with no type annotations whatsoever. In practice, however, Haskell programs contain many userwritten type signatures, for two main reasons.

First, the type of a function can be extremely helpful as documentation, with the advantage that it is machine-checked documentation. Almost all programmers regard it as good practice to provide a signature for every top-level function. Indeed, GHC has a warning flag, -Wmissing-signatures, which enforces this convention.

Second, as Haskell's type system becomes increasingly expressive, complete type inference becomes intractable, and the type system necessarily relies on programmer-supplied type annotations. Here are some examples:

- Type-class ambiguity is present even in Haskell 98. Consider ${ }^{1}$ :

normalize :: String $\rightarrow$ String

normalize $s=$ show $($ read $s)$

This function parses a string to a value of some type, and then turns that value back into a string. But nothing in the code specifies that type, so the programmer must disambiguate. One way to do so is to provide a type signature that specifies the result type of the read $s$ call, thus:

normalize $s=$ show $($ read $s:: \ln t)$

- Polymorphic recursion. In ML, recursive calls to a function must be at monomorphic type, but Haskell has always supported polymorphic recursion, provided the function has a type signature. For example:

data $T$ a = Leaf a $\mid$ Node $(T[a])(T[a])$

leaves : $T a \rightarrow[a]$

leaves $($ Leaf $x)=[x]$

leaves $($ Node $t 1 \mathrm{t} 2)=$ concat $($ leaves $t 1+$ leaves $t 2)$

- Higher-rank types [Peyton Jones et al. 2007]. Consider

$f::(\forall a .[a] \rightarrow[a]) \rightarrow([$ Char $],[$ Bool $])$

$f g=(g$ "Hello", $g[$ True, False $])$

\footnotetext{
${ }^{1}$ Figure 1 gives the types of standard functions, such as read and show.
}

Here the type of $g$ is polymorphic, so it can be applied to lists of different type. The type signature is essential to specify the type of the argument $g$; without it, $f$ will be rejected.

- Generalized algebraic data types [Schrijvers et al. 2009]. The popular GADTs extension to GHC allows pattern matching to refine the type information available in the right hand side of an equation. Here is an example:

$$
\begin{aligned}
& \text { data } G \text { a where } \\
& \text { MkInt }:: G \text { Int } \\
& \text { MkFun }:: G(\ln t \rightarrow \operatorname{Int})
\end{aligned}
$$

When we learn that a value $g$ :: $G a$ is actually the constructor MkInt, then we simultaneously learn that $a$ really is Int. GHC can use this fact during type checking the right-hand side of a function, like this:

$$
\begin{aligned}
& \text { match } G:: G a \rightarrow a \\
& \text { matchG MkInt }=5 \\
& \text { matchG MkFun }=(+10)
\end{aligned}
$$

Again, however, match $G$ will only type-check if it is given a signature; see Schrijvers et al. [2009] for details.

- Ambiguous types. Consider

$$
\begin{aligned}
& \text { type family } F a \\
& \text { ambig :: Typeable } a \Rightarrow F a \rightarrow \text { Int } \\
& \text { test }:: \text { Char } \rightarrow \text { Int } \\
& \text { test } x=\text { ambig } x
\end{aligned}
$$

In test GHC must decide at what type to call ambig; that is, what type should instantiate the $a$ in ambig's type. Any choice $a=\tau$ must ensure that $F \tau \sim$ Char but, because $F$ might not be injective, that does not tell us what $a$ should be. A type signature is not enough to resolve this case; we need a different form of type annotation, namely visible type application (Section 2.3).

There is a general pattern here: as the type system becomes more expressive, the type inference needs more guidance. Moreover, that guidance is extremely informative to the programmer, as well as to the compiler.

\subsection{Support for Scoped Type Variables}

Given the increasing importance of type annotations, a good principle is this: it should be possible to specify, via a type signature, the type of any sub-expression or any let-binding. Alas, as shown in the introduction, Haskell98 supports only closed type signatures, so there are useful type signatures that we simply cannot write.

The key deficiency in Haskell98 is that it provides no way to bring type variables into scope. GHC has recognized this need for many years, and the ScopedTypeVariables extension offers two ways to bring a type variable into scope:

- Binding in a declaration signature. Since 2004 GHC allows you to write 


$$
\begin{gathered}
\text { prefix }:: \forall a . a \rightarrow[[a]] \rightarrow[[a]] \\
\text { prefix } x \text { yss }=\text { map xcons yss } \\
\text { where xcons }::[a] \rightarrow[a] \\
\text { xcons ys }=x: y s
\end{gathered}
$$

The explicit " $\forall$ " brings $a$ into scope in the rest of the type signature (of course), but it also brings a into scope in the body of the named function, prefix in this case. The rule is a bit strange, because the definition of prefix is not syntactically "under" the $\forall$, and indeed the signature can be written far away from the actual binding. But in practice the rule works well, and we take it as-is for the purposes of this paper.

- Pattern signatures: binding a type variable in a pattern. For even longer, since 1998, GHC has allowed you to write this:

$$
\begin{gathered}
\text { prefix }(x:: b) \text { yss }=\text { map xcons yss } \\
\text { where } x \text { cons }::[b] \rightarrow[b] \\
x \text { cons } y s=x: y s
\end{gathered}
$$

Here, we bind the type variable $b$ in the pattern $(x:: b)$, and this binding scopes over the body of the binding. We describe pattern signatures in much more detail in Section 3.

\subsection{Visible Type Application}

The TypeApplications extension provides a relatively new form of type annotation: explicit type applications, first described by Eisenberg et al. [2016]. The idea is that an argument of the form @ty specifies a type argument. This can often be used more elegantly than a type signature. For example, a hypothetical unit-test for the function isJust :: Maybe $a \rightarrow$ Bool,

$$
\text { testIsJust } 1=\text { isJust }(\text { Just }(2018:: \text { Int })) \quad==\text { True }
$$$$
\text { testlsJust } 2=\text { isJust }(\text { Nothing }:: \text { Maybe Int })==\text { False }
$$

can equivalently be written more elegantly using explicit type annotations

testIsJust $1=$ isJust $($ Just @ Int 2018) $==$ True
testlsJust $2=$ isJust $($ Nothing @Int $)==$ False

Visible type application solves the awkward case of ambig in Section 2.1: we can disambiguate the call with a type argument. For example:

type family $F a$

type instance $F$ Bool $=$ Char

ambig :: Typeable $a \Rightarrow F a \rightarrow$ Int

test :: Char $\rightarrow$ Int

test $x=$ ambig @Bool $x$

Here we specify that ambig should be called at Bool, and that is enough to type-check the program.

It is natural to wonder whether we can extend visible type application to patterns, just as we extended type signatures to patterns. Doing so is the main language extension suggested in this paper: Section 4.

\section{Pattern Signatures and Their Shortcomings}

We see above that ScopedTypeVariables enables the user to bind type variables in patterns, by providing a pattern signature, that is, a type signature in a pattern. We explore pattern signatures and their shortcomings in this section.

\subsection{The Binding Structure of a Pattern Signature}

A pattern signature may bind a type variable, but it may also mention a type variable that is already in scope. For example, we may write

$$
\begin{array}{r}
\text { prefix }(x:: a) \text { yss }=\text { map xcons yss } \\
\text { where xcons }(y s::[a])=x: y s
\end{array}
$$

Here, the pattern signature $(x:: a)$ binds $a$ (as well as $x$ ), but the pattern signature (ys:: $[a]$ ) simply mentions $a$ (which is already in scope), as well as binding ys. The rule is this: a use of a type variable $p$ in a pattern signature is an occurrence of $p$ if $p$ is already in scope; but binds $p$ if $p$ is not already in scope.

It is entirely possible to have many different type variables in scope, all of which are aliases for the same type. For example:

$$
\begin{aligned}
& \text { prefix }:: \forall a . a \rightarrow[[a]] \rightarrow[[a]] \\
& \text { prefix }(x:: b)(y s s::[[c]])=\text { map xcons yss } \\
& \quad \text { where xcons }(y s::[d])=x: y s
\end{aligned}
$$

Here $a, b, c$, and $d$ are all in scope in the body of xcons, and are all aliases for the same type.

The current implementation of ScopedTypeVariables allows such lexically-scoped type variables to stand only for other type variables, and not for arbitrary types, a point we return to in Section 3.5.

\subsection{Pattern Signatures Are Useful}

Pattern signatures have merit even if there are no type variables around. Consider this Haskell program:

$$
\begin{aligned}
& \text { main }=\text { do } x \leftarrow \operatorname{read} L n \\
& \text { if null } x \text { then putStrLn "Empty" } \\
& \text { else putStrLn "Not empty" }
\end{aligned}
$$

The types of the program are ambiguous: Clearly, $x$ is some type that has a Read instance, and because it is passed to null, is a list ${ }^{2}$, but the compiler needs to know the precise type, and rejects the program.

To fix this in Haskell98, the programmer has two options:

\footnotetext{
${ }^{2}$ Or, with a recent version of the standard library, it is something with a Foldable instance.
} 
- They can wrap the call to readLn in a type annotation:

$$
\begin{gathered}
\text { main }=\text { do } x \leftarrow(\text { readLn }:: I O[\operatorname{Int}]) \\
\text { if null } x \text { then putStrLn "Empty!" } \\
\text { else print } x
\end{gathered}
$$

but this is infelicitous because there is no question that read $L n$ is in the $I O$, and with larger types this can get very verbose.

- They can wrap an occurrence of $x$ in a type annotation:

$$
\begin{aligned}
& \text { main }=\text { do } x \leftarrow \text { readLn } \\
& \text { if null }(x::[\operatorname{lnt}]) \text { then putStrLn "Empty" } \\
& \text { else print } x
\end{aligned}
$$

but again this is unsatisfying, because it feels too late.

Both variants are essentially work-arounds for the natural way of specifying the type of $x$, namely at its binding site:

$$
\begin{gathered}
\text { main }=\text { do }(x::[\ln t]) \leftarrow \text { readLn } \\
\text { if null } x \text { then putStrLn "Empty" } \\
\text { else print } x
\end{gathered}
$$

which is precisely what the PatternSignatures language extension provides ${ }^{3}$-the ability to write a type annotation in a pattern.

Users, especially beginners, who have to track down a confusing type error in their code, can now exhaustively type annotate not just their terms, but also their patterns, until they have cornered the bug.

\subsection{Pattern Signatures Are Essential}

Pattern signatures become more crucial when we consider existential types. The ExistentialQuantification extension allows users to bind existential variables in their data constructors. These are type variables whose values are "stored" by a constructor (but not really, because types are erased) and made available during pattern matching. Here are two examples:

$$
\begin{aligned}
& \text { data Ticker where } \\
& \text { MkTicker : } \forall a . a \rightarrow(a \rightarrow a) \rightarrow(a \rightarrow \text { Int }) \rightarrow \text { Ticker } \\
& \text { data Showable where } \\
& \quad \text { MkShowable : } \forall a \text {. Show } a \Rightarrow a \rightarrow \text { Showable }
\end{aligned}
$$

A Ticker contains an object of some type (but we do not know what type), along with an update function of type $a \rightarrow a$ and a way to convert an $a$ into an Int. The Showable type packs a value of an arbitrary type that has a Show instance along with its Show dictionary. Here are some functions that operate on these types:

-- Updates a ticker, returning whether or not the ticker

-- has reached a limit

$$
\text { tick }:: \text { Ticker } \rightarrow \text { Int } \rightarrow \text { (Ticker, Bool) }
$$

\footnotetext{
${ }^{3}$ Modern GHC actually folds PatternSignatures into ScopedTypeVariables, giving both extensions the same meaning. However, it is expositionally cleaner to separate the two, as we do throughout this paper.
}

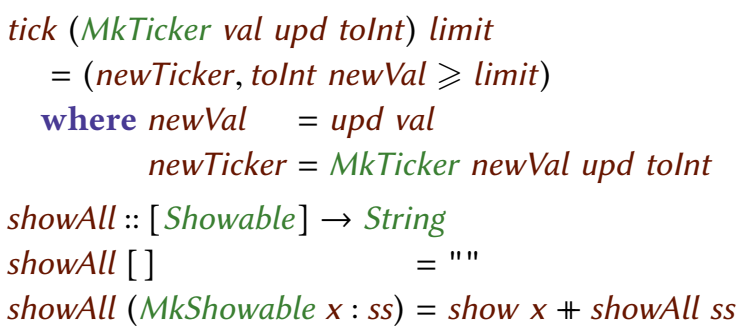

We see that the tick function can unpack the existential in the Ticker value and operate on the value of type $a$ without ever knowing what $a$ is. Similarly, the showAll function works with data of type $a$ knowing only that $a$ has a Show instance. (The Show a constraint is brought into scope by the pattern match.)

However, existentials can never escape, forbidding the following function:

jailbreak (MkTicker val _ _ $)=$ val

What should the type of jailbreak be? There is no answer to this question (jailbreak :: Ticker $\rightarrow a$ is clearly too polymorphic), and so GHC rejects this definition, correctly stating that type variable 'a' would escape its scope.

We naturally wish to name the existential type variable sometimes. For example, suppose we wanted to give new Val (in the where clause of tick) a type signature. Saying newVal:: $a$ would hardly do, because there is not yet a connection between the name $a$ and the type unpacked from MkTicker. We have to do this:

$$
\begin{aligned}
& \text { tick (MkTicker (val :: b) upd tolnt) limit }=\ldots \\
& \text { where newVal :: } b \\
& \text { newVal = upd val }
\end{aligned}
$$

The $v a l:: b$ in the pattern binds the type variable $b$, so we can refer back to it later.

\subsection{Pattern Signatures Are Clumsy}

Pattern signatures can be clumsy to use, when the type variable is buried deep inside an ornate type. Here is a contrived example:

$$
\begin{aligned}
& \text { data Elab where } \\
& \quad \text { MkElab :: Show } a \Rightarrow[\text { Maybe }(\operatorname{Tree}(a, \ln t))] \rightarrow \text { Elab } \\
& \text { getE }:: \text { Elab } \rightarrow \text { Int } \\
& \text { getE }(\text { MkElab }(x s::[\text { Maybe }(\operatorname{Tree}(a, \operatorname{Int}))]))=\ldots a \ldots
\end{aligned}
$$

To bring $a$ into scope in $f$ 's right-hand side, we have to repeat the MkElab's elaborate argument type.

More seriously, it may be impossible, rather than merely clumsy, to bind the variable we need. Consider the following GADT:

$$
\begin{aligned}
& \text { data GM a where } \\
& \qquad \text { MkMaybe : GM (Maybe } b) \\
& \text { matchGM :: } a \rightarrow G M a \rightarrow \text { Bool } \\
& \text { matchGM } \text { x MkMaybe }=\text { isJust } x
\end{aligned}
$$


This definition works just fine: GHC learns that $x$ 's type is Maybe $b$ (for some existential $b$ ) and the call to isJust is well typed. But what if we want to bind $b$ in this definition? Annoyingly, MkMaybe has no argument to which we can apply a pattern signature. Nor does it work to wrap a pattern signature around the outside of the match, thus:

matchGM :: $a \rightarrow$ GM $a \rightarrow$ Bool

matchGM x (MkMaybe :: GM (Maybe b)) = isJust @bx

This definition is rejected. The problem is that the type annotation on the MkMaybe pattern is checked before the pattern itself is matched against ${ }^{4}$. Before matching the MkMaybe, we do not yet know that $a$ is really Maybe $b$. Nor can we put the type annotation on $x$, as that, too, occurs before the MkMaybe pattern has been matched. A possible solution is this monstrosity:

$$
\begin{aligned}
& \text { matchGM :: } a \rightarrow G M a \rightarrow \text { Bool } \\
& \text { matchGM x gm@MkMaybe = case gm of } \\
& \quad\left(\_:: G M(\text { Maybe b) }) \rightarrow\right. \text { isJust @bx }
\end{aligned}
$$

This is grotesque. Of course, in this case, we can simply flip the argument order to match $G M$, but we should not be forced to change argument order just because of clumsy syntax. We must do better, and we will in Section 4 .

\subsection{Pattern Signatures Resist Refactoring}

In Section 3.1, we explained that a scoped type variable may refer only to another type variable. This means that the definition

$$
\begin{aligned}
& \text { prefix }:: a \rightarrow[[a]] \rightarrow[[a]] \\
& \text { prefix }(x:: b) \text { yss }=\text { map xcons yss } \\
& \text { where xcons ys }=x: y s
\end{aligned}
$$

is accepted, because $b$ stands for the type variable in the type of prefix. But suppose, for example, we specialize the type signature of prefix without changing its definition:

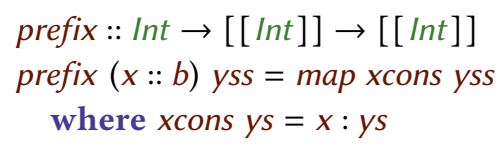

Now this definition is rejected with the error message "Couldn't match expected type $b$ with actual type Int", because $b$ would have to stand for Int.

Since the design of ScopedTypeVariables, GHC has evolved, and with the advent of type equalities, the restriction itself becomes confusing. Should GHC accept the following definition?

$$
\begin{aligned}
& \text { prefix }:: a \sim \operatorname{Int} \Rightarrow \operatorname{Int} \rightarrow[[a]] \rightarrow[[a]] \\
& \text { prefix }(x:: b) \text { yss }=\operatorname{map} x \text { cons yss } \\
& \text { where xcons ys }=x: y s
\end{aligned}
$$

\footnotetext{
${ }^{4}$ This ordering arises because any type variable in a pattern signature is bound within the pattern.
}

Is $b$ an alias for $a$ (legal) or for Int (illegal)? Since $a$ and Int are equal, the question does not really make sense. We therefore propose to simply drop this restriction (Section 4.3).

\subsection{Pattern Signatures Are Inadequate}

We end our growing list of infelicities with a case in which there is no way whatsoever to bind the type variable, short of changing the data type definition.

Type families [Chakravarty et al. 2005; Eisenberg et al. 2014] allow users to write type-level functions and encode type-level computation. For example, we might write this:

type family $F a$ where

$$
\begin{aligned}
& F \text { Int }=\text { Bool } \\
& F \text { Char }=\text { Double } \\
& \text { F Float }=\text { Double }
\end{aligned}
$$

Naturally, we can use a type family to define the type of an argument to an existential data constructor:

data $T F$ where

$$
\text { MkTF :: } \forall a \text {. Typeable } a \Rightarrow F a \rightarrow T F
$$

The MkTF constructor stores a value of type $(F a)$; it also stores a dictionary for a Typeable a constraint [Peyton Jones et al. 2016] - that is, we can use a runtime type test to discover the choice for $a$. We would thus like to write the following function:

$$
\begin{aligned}
& \text { toDouble }:: \text { TF } \rightarrow \text { Double } \\
& \text { toDouble }(\text { MkTF } x) \text {-- We expect } x:: F \text { a } \\
& \text { | Just HRefl } \leftarrow \text { isType @Int }=\text { if } x \text { then } 1.0 \text { else }-1.0 \\
& \text { | Just HRefl } \leftarrow \text { isType @Char }=x \\
& \text { | Just HRefl } \leftarrow \text { isType @Float }=x \\
& \text { | otherwise }=0.0 \\
& \text { where } \\
& \text { isType }:: \forall t y . \text { Typeable ty } \Rightarrow \text { Maybe }(a: \approx: t y) \\
& \text { isType }=\text { eqTypeRep (typeRep @a) (typeRep @ty) }
\end{aligned}
$$

The specifics of this function are not important here (see [Peyton Jones et al. 2016]). For our present purposes, the crucial point is this: the existentially-bound type $a$ is mentioned in both the definition of isType and its type signature-but there is no way to bring a into scope. We might try using a pattern signature at the binding of $x$, thus:

toDouble $(\operatorname{MkTF}(x:: F$ a $))=\ldots$

but that does not quite work. The problem is that $F$ is not injective. The $a$ in that pattern type annotation need not be the same one packed into the existential type variable by MkTF, and GHC rightly considers such an $a$ to be ambiguous ${ }^{5}$.

The only workaround available today is to change MkTF to take a proxy argument:

\footnotetext{
${ }^{5}$ If $F$ were an injective type family, we could label it as such to fix the
} problem [Stolarek et al. 2015]. But here we assume that it is not. 


$$
\begin{aligned}
& \text { data Proxy } a=\text { Proxy } \quad-\text { in GHC’s Data.Proxy } \\
& \text { data TF where } \\
& \text { MkTF :: } \forall a \text {. Typeable } a \Rightarrow \text { Proxy } a \rightarrow F a \rightarrow T F \\
& \text { toDouble }(\text { MkTF }(\text { _: Proxy } a) x)=\ldots
\end{aligned}
$$

The Proxy type stores no runtime information (at runtime, it is isomorphic to ()), but the type Proxy a carries the allimportant type variable $a$. All datatypes are injective, so we can use this proxy argument to bind the type variable $a$ in a way that we could not do previously.

As with many other examples, this is once again unsatisfying: it is a shame that we have to modify the data constructor declaration just to deal with type variable binding.

\subsection{Conclusion}

In this section we have seen that pattern signatures allow us to bring into scope the existentially-bound type variables of a data constructor, but that doing so can be clumsy, and occasionally impossible. We need something better.

\section{Visible Type Application in Patterns}

Consider again Elab from Section 3.4:

$$
\begin{aligned}
& \text { data Elab where } \\
& \qquad \text { MkElab:: Show } a \Rightarrow[\text { Maybe }(\text { Tree }(a, \ln t))] \rightarrow \text { Elab }
\end{aligned}
$$

and suppose we want to build a value of type Elab containing an empty list. We cannot write just MkElab [] because that is ambiguous: we must fix the type at which MkElab is called so that the compiler can pick the right Show dictionary. We can use a type signature, but it is clumsy, just as the pattern signature was clumsy in Section 3.4:

\section{MkElab ([]:: [Maybe $($ Tree $($ Bool, Int $))])$}

It is much nicer to use visible type application and write MkElab@Bool []. So it is natural to ask whether we could do the same in patterns, like this:

getE :: Elab $\rightarrow$ Int

$\operatorname{get} E($ MkElab@axs) =...a...

Here, we bind $a$ directly ${ }^{6}$, as a type-argument pattern all by itself, rather than indirectly via a pattern signature.

We call this visible type application in patterns, a dual of visible type application in the same way that a pattern signatures are a dual of type signatures. This section describes visible type application in patterns informally, while the next formalizes it.

\footnotetext{
${ }^{6}$ One might reasonably wonder how we can steal @ in a pattern in this way. After all, Haskellers can also write, e.g., $f$ list@ $(x: x s)=\ldots$ to alias list to the pattern $(x: x s)$. The new syntax is not actually ambiguous, however: an as-pattern always has a variable on its left, while our new form is always headed by a data constructor with all type patterns preceding value-level patterns.
}

This feature was first requested more than two years ago.? Furthermore, binding type variables like this is useful for more than just disambiguation, as we will shortly see.

\subsection{Examples}

Visible type application in patterns immediately fixes the other problems of pattern signatures identified above. For example, in the GADT example of Section 3.4 we can write matchGM :: $a \rightarrow$ GM $a \rightarrow$ Bool matchGM x (MkMaybe @b) = isJust @bx and for the type-family example of Section 3.6 we write toDouble $($ MkTF @a $\mathrm{x})=.$.

\subsection{Universal and Existential Variables}

Visible type applications in patterns can be used for all the type arguments of a data constructor, whether existential or universal. As an example of the latter we may write

$$
\begin{gathered}
\text { main }=\text { do }(\text { Just } @ I n t x) \leftarrow \text { readMaybe 'fmap' getLine } \\
\text { putStrLn "Input was " \# show } x
\end{gathered}
$$

as an alternative to

$$
\begin{gathered}
\text { main }=\text { do }(\text { Just }(x:: \text { Int })) \leftarrow \text { readMaybe 'fmap' getLine } \\
\text { putStrLn } \$ \text { "Input was " }+ \text { show } x
\end{gathered}
$$

Visible type application in patterns considers the type of data constructor, exactly as written by the user. For example

$$
\begin{aligned}
& \text { data } G a b \text { where } \\
& \text { G1 :: } \forall b . \text { Char } \rightarrow G \text { Int } b \\
& G 2:: \forall p q b . p \rightarrow q \rightarrow b \rightarrow G(p, q) b \\
& G 3:: \forall p q a b .(a \sim(p, q)) \Rightarrow p \rightarrow q \rightarrow b \rightarrow G a b \\
& f:: \text { G a Bool } \rightarrow \text { Int } \\
& f(G 1 @ \text { Bool } y) \quad=\text { ord } y \\
& f(G 2 @ p @ q x y z)=0 \\
& f(G 3 @ p @ q @ a @ B o o l x y z)=1
\end{aligned}
$$

\subsection{Type Aliases}

In Section 3.5 we have seen that GHC currently restricts type variables to refer to type variables, but that this does not have to be the case. Similar questions arise in our function $f$ above. Could we write this for G3?

\section{$f(G 3 @ p @ q @(p, q) @ b x y z)=1$}

\footnotetext{
${ }^{7}$ https://ghc.haskell.org/trac/ghc/ticket/11350
} 


$\begin{array}{lcl}\text { Term variables } & \ni x, y, z, f, g, h \\ \text { Internal type vars } & \ni a, b \\ \text { User type vars } & \ni c & \\ \text { Data constructors } & \ni \mathrm{K} \\ \text { Atoms } & v::=\mathrm{K} \mid x \\ \text { Expressions } & e::=v|\lambda x \cdot e| e_{1} e_{2} \mid \text { case } e \text { of }\{\overline{p \rightarrow e}\} \\ & \mid \text { let } x:: \tau=e_{1} \text { in } e_{2} \\ \text { Patterns } & p::=x \mid \mathrm{K} \bar{p} \\ \text { Polytypes } & \sigma::=\forall \bar{a} \cdot \tau \\ \text { Monotypes } & \tau, v::=t v|\mathrm{~T} \bar{\tau}| \ldots \\ \text { Type variables } & t v::=a \mid c \\ \text { Type env } & \Gamma::=\epsilon \mid \Gamma, v: \sigma \\ \text { Substitutions } & \theta::= & {[\overline{t v \mapsto \tau}]} \\ \text { Types of data cons } & \Gamma_{t}= & \overline{\mathrm{K}: \forall \bar{a} \cdot \bar{\sigma} \rightarrow \mathrm{T} \bar{a}}\end{array}$

Figure 2. The initial grammar

Instead of $a$ we have written $(p, q)$, which is equal to $a$. And instead of Bool we have written $b$, thereby binding $b$ to Bool.

Given the ubiquity of equalities, it no longer seems to make sense to restrict what a scoped type variable can stand for, so we propose simply to drop the restriction. Doing so simplifies the specification and the implementation of both pattern signatures and visible type application in patterns. A GHC proposal by one of the authors [Breitner 2018] is underway. Relaxing the requirement also allows the user to use type variables as "local type synonyms" that stand for possibly long types:

processMap :: Map Int (Maybe (Complex Type)) $\rightarrow$... processMap $(m::$ Map key value $)=\ldots$

\section{Formal Specification}

We give the first formal specification of a number of extensions to Haskell related to pattern matching and the scoping of type variables: annotations in patterns, scoped type variables, and type application syntax in patterns. This section builds up these specification step by step, starting with a specification of the language without these features.

Our specification does not cover let-bindings and declaration type signatures. We focus instead on pattern signatures, which is where our new contribution lies. The scoping of forall-bound type variables from declaration type signatures would be straightforward to add.

\subsection{The Baseline}

We begin with a reduced model of Haskell98 terms, which knows nothing yet about scoped type variables nor type equalities (i.e., no GADTs). We also removed type class constraints. The syntax is given in Fig. 2, and the typing rules are in Fig. 3. In the typing rules, we use a convention where an over-bar indicates a list, optionally with a superscript index to indicate the iterator. Iterators are additionally annotated with length bounds, where appropriate.

The grammar includes separate metavariables for internal type variables $a$ and user type variables $c$. The former are type variables as propagated by the compiler, while the latter are type variables the user has written. It is as if internal type variables $a$ are spelled with characters unavailable in source Haskell. This distinction becomes important in Section 5.5. The language also includes only annotated let-bindings; no let-generalization here. (The "generalization" you might spot in Rule LET is simply quantifying over the variables the user has lexically written in the type signature.) This keeps our treatment simple and avoids the challenges of type inference. Allowing full let-generalization and un-annotated lets changes none of the conclusions presented here.

The judgment $\Gamma \vdash e: \tau$ indicates that the term $e$ has type $\tau$ in the context $\Gamma$, where $\Gamma$ is a list of term variables and their (possibly polymorphic) types. Data constructors are globally fixed in an initial top-level context $\Gamma_{t}$; it is assumed that any context $\Gamma$ contains the global $\Gamma_{t}$ binding data constructors.

The type-checking of possibly nested patterns, as they occur in a case statement, is offloaded to the judgment $\Gamma \vdash_{p} p$ : $\sigma \Rightarrow \Gamma^{\prime}$, which checks that $p$ is a pattern for a value of type $\sigma$ and possibly binds new term variables, which are added to $\Gamma$ and returned in the extended environment $\Gamma^{\prime}$. The auxiliary judgment $\Gamma r_{\mathrm{p}}^{*}{\overline{p_{i}: \sigma_{i}}}^{i} \Rightarrow \Gamma^{\prime}$ straightforwardly threads the environment through a list of such pattern typings.

These rules should be unsurprising, but provide a baseline from which to build.

\subsection{Support for GADTs}

Now we extend this language with support for GADTs, with their existential type variables and equality constraints. See Fig. 4. The term syntax is unchanged, but polytypes now can mention constraints, which can either be empty (and elided from this text), an equality between two monotypes, or a conjunction of constraints. We leave the possibility open for additional constraints, as indicated by the ellipsis.

The environment $\Gamma$ is extended with two new forms. First, we track the scope of type variables by adding $a: *$ to $\Gamma^{8}$. Second, we add constraints $Q$ to $\Gamma$, to indicate which constraints (bound by a GADT pattern match) are in scope. Conversely, constraints are proved by the $\Gamma \Vdash Q$ entailment relation. As type inference and entailment is not the subject of this paper, we leave this relation abstract. The concrete instantiation of this judgment by, e.g., that of Vytiniotis et al.

\footnotetext{
${ }^{8}$ Haskell supports higher kinds, but we elide that here for simplicity, and assume that all type variables have kind $*$.
} 


$$
\begin{aligned}
& \Gamma \vdash e: \tau \quad \text { Expression typing } \\
& \frac{\Gamma, x: \tau_{1} \vdash e: \tau_{2}}{\Gamma \vdash \lambda x . e: \tau_{1} \rightarrow \tau_{2}} \text { ABS } \quad \frac{\Gamma \vdash e_{1}: \tau_{1} \rightarrow \tau_{2} \quad \Gamma \vdash e_{2}: \tau_{2}}{\Gamma \vdash e_{1} e_{2}: \tau_{2}} \text { APP } \quad \begin{array}{l}
\Gamma \vdash e: v \quad \bar{c}=f t v(v) \\
\Gamma, x: \forall \bar{c} . v \vdash e_{2}: \tau
\end{array}
\end{aligned}
$$

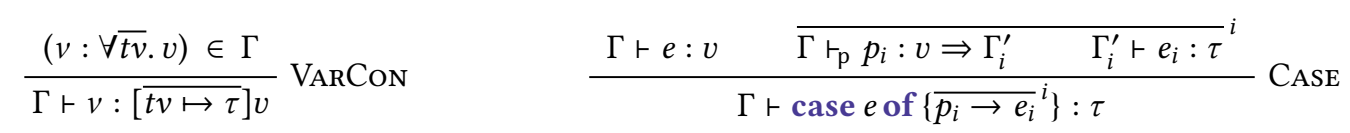

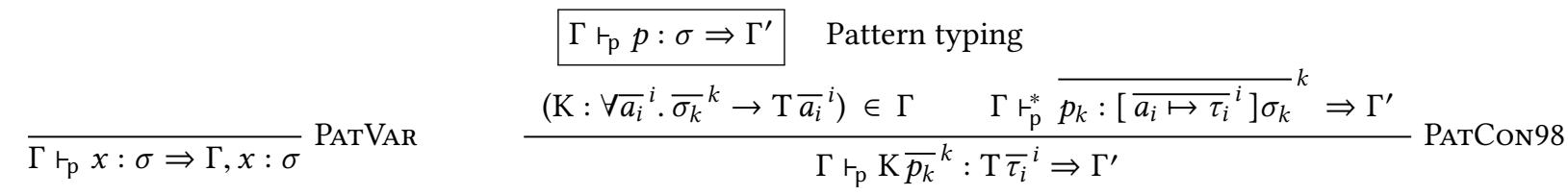

$$
\begin{aligned}
& \Gamma r_{\mathrm{p}}^{*}{\overline{p_{i}: \sigma_{i}}}^{i} \Rightarrow \Gamma^{\prime} \quad \text { Pattern sequence typing }
\end{aligned}
$$

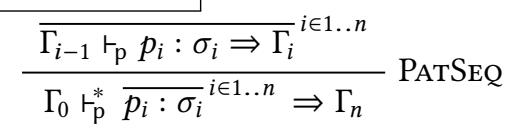

Figure 3. Typing of Haskell98 patterns

$$
\begin{aligned}
& \text { Constraints } \\
& \text { Polytypes } \\
& \text { Type env } \\
& \text { Types of data cons } \\
& \text { Constraint entailment } \\
& \Gamma \vdash e: \tau \quad \text { Expression typing } \\
& \begin{array}{ll}
(v: \forall \bar{a} . Q \Rightarrow v) \in \Gamma & \Gamma \vdash e: \tau_{1} \\
\frac{\Gamma \Vdash[\overline{a \mapsto \tau}] Q}{\Gamma \vdash v:[\overline{a \mapsto \tau}] v} \text { VARCoNQ } & \frac{\Gamma \Vdash \tau_{1} \sim \tau_{2}}{\Gamma \vdash e: \tau_{2}} \text { EQ }
\end{array} \\
& \overline{\Gamma \vdash \mathrm{p}}_{p_{i}: v \Rightarrow \Gamma_{i}^{\prime} \quad \Gamma_{i}^{\prime} \vdash e_{i}: \tau}{ }^{i} \\
& \frac{\Gamma \vdash e: v \quad f t v(\tau) \subseteq \operatorname{dom}(\Gamma)}{\Gamma \vdash \text { case } e \text { of }\left\{{\overline{p_{i} \rightarrow e_{i}}}^{i}\right\}: \tau} \text { CASETV }
\end{aligned}
$$

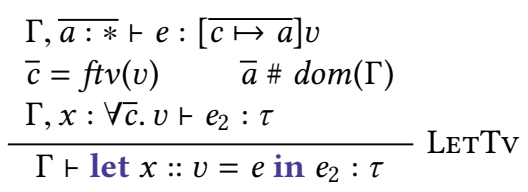

$$
\begin{aligned}
& \Gamma \vdash_{\mathrm{p}} p: \sigma \Rightarrow \Gamma^{\prime} \quad \text { Pattern typing } \\
& \left(\mathrm{K}: \forall \bar{a} . Q \Rightarrow{\overline{\sigma_{i}}}^{i} \rightarrow \mathrm{T}{\overline{v_{j}}}^{j}\right) \in \Gamma \quad \bar{a} \# \operatorname{dom}(\Gamma) \\
& \Gamma, \overline{a: *}_{v_{j} \sim \tau_{j}}^{j}, Q \vdash_{\mathrm{p}}^{*}{\overline{p_{i}: \sigma_{i}}}^{i} \Rightarrow \Gamma^{\prime} \\
& \Gamma \vdash_{\mathrm{p}} \mathrm{K}{\overline{p_{i}}}^{i}: \mathrm{T}{\overline{\tau_{j}}}^{j} \Rightarrow \Gamma^{\prime}
\end{aligned}
$$

Figure 4. Adding support for GADTs
[2011] would be appropriate in an implementation of this type system.

Support for GADTs can be seen in the new form of data constructor types, listed in Fig. 4. Note that the arguments to $\mathrm{T}$ in the return type are no longer confined to be $\bar{a}$, the quantified type variables; instead they can be arbitrary monotypes. In addition, a constructor can include a constraint $Q$.

When a data constructor is used in an expression, then the type equalities must be satisfied in the current environment, as expressed by the new premise of Rule VARCONQ in Fig. 4. We see also that the type equalities in the environment can be used for implicit coercion, as expressed in the Rule EQ.

When pattern-matching a data constructor, Rule PATCON brings the type variables $\bar{a}$ into scope, by extending $\Gamma$. We require that these bound variables are fresh with respect to other variables in scope, a requirement we can satisfy by $\alpha$-renaming if necessary. We also add the type equalities that we have learned to the environment-that is, the equivalence between the ${\overline{v_{j}}}^{j}$ from the data constructor's type and the ${\overline{\tau_{j}}}^{j}$ from the pattern type.

Finally, we update Rule CASETv to prevent skolem escape and Rule LETTV to track the internal variables brought into scope. Note that these variables are internal only-the user cannot write them in a program.

At this point, our type system is comparable in expressiveness to the specification given by Vytiniotis et al. [2011]. A notable difference is that we explicitly handle nested patterns. This is important, as in the presence of GADTs, the precise formulation of how nested pattern are type-checked matters. For example, consider: 


$$
\begin{aligned}
& \text { data } G \text { a where } \\
& \text { G1 :: G Bool } \\
& \text { G2 :: G a } \\
& f::(G a, a, G a) \rightarrow \text { Bool } \\
& f(G 1, \text { True },-)=\text { False } \\
& f(\rightarrow, \text { True }, G 1)=\text { False }
\end{aligned}
$$

Here the first equation for $f$ is fine, but the second is not, because the pattern True cannot match against an argument of type $a$ until after the constructor $G 1$ has been matchedand matching in Haskell is left-to-right.

\subsection{Treating Universals and Existentals Uniformly}

A technical contribution of this paper is that Rule PATCON is simpler and more uniform than the one usually given [e.g. by Vytiniotis et al. 2011], in that it does not distinguish the universal and existential type variables of the data constructor. Instead, all the type variables are freshly bound, with the equalities ${\overline{v_{j} \sim \tau_{j}}}^{j}$ linking them to the context. In particular, these equalities take the place of the substitution written in the previous Rule Con98.

However, there is a worry: pattern-matching involving GADTs lacks principal types, and hence usually requires a type signature (see Section 2.1). If we treat vanilla, nonGADT Haskell98 data types in the same way as GADTs, do we lose type inference for ordinary Haskell98 definitions? Specifically, Vytiniotis et al. [2011, Section 5.6.1] describe how assumed local constraints can interfere with type inference, essentially by making certain unification variables "untouchable" (that is, unavailable for unification). That section also describes how to make more unification variables touchable in the non-GADT case, when the constraints entail no equalities. But our typing rule introduces equalities even in the non-GADT case, so this mechanism fails for us.

Let us investigate Rule PATCON specialized to the case of an ordinary, non-GADT constructor, which binds no context $Q$ and does not constrain its result type arguments:

$$
\frac{\left(\mathrm{K}: \forall{\overline{a_{j}}}^{j} \cdot{\overline{\sigma_{i}}}^{i} \rightarrow \mathrm{T}{\overline{a_{j}}}^{j}\right) \in \Gamma \quad \bar{a} \# \operatorname{dom}(\Gamma)}{\Gamma,{\overline{a_{j}}: *}^{j},{\overline{a_{j}} \sim \tau_{j}^{j}}^{j}{\mathrm{r}_{\mathrm{p}}^{*}}^{i}{\overline{p_{i}}}^{i} \Rightarrow \Gamma^{\prime}}
$$

We see that all of its assumed equality constraints take the form $a_{j} \sim \tau_{j}$, where $a_{j}$ is freshly bound. We can view such equalities not as true assumed equalities (which lead to the type inference problems for GADTs), but instead as a form of local let-binding: the context simply gives us the definition of these type variables. In this interpretation, it is critical that the type variable in the equality assumption is freshly bound-that is, we are not referring to a type variable from a larger scope. Viewing the equalities in $\Gamma^{\prime}$ as let-like, it is sensible to extend the ad-hoc extension of Vytiniotis et al. [2011] to include such forms. Indeed, doing so is an independently-useful improvement to type inference, and
Patterns $\quad p::=\ldots \mid p:: \sigma$

$\frac{f t v\left(\sigma^{\prime}\right)=\emptyset \quad \Gamma \Vdash \sigma \leq \sigma^{\prime} \quad \Gamma \vdash_{\mathrm{p}} p: \sigma^{\prime} \Rightarrow \Gamma^{\prime}}{\Gamma \vdash_{\mathrm{p}}\left(p:: \sigma^{\prime}\right): \sigma \Rightarrow \Gamma^{\prime}}$ PATSIG

Figure 5. Syntax and typing rule for pattern signatures

$$
\begin{aligned}
& \text { Expressions } \\
& e::=\ldots \mid \text { let } x:: \sigma=e_{1} \text { in } e_{2} \\
& \sigma=\forall \bar{c} . Q \Rightarrow v \quad f t v(\sigma) \subseteq \operatorname{dom}(\Gamma) \\
& \frac{\Gamma, \overline{c: *}, Q \vdash e: v \quad \Gamma, x: \sigma \vdash e_{2}: \tau}{\Gamma \vdash \text { let } x:: \sigma=e \text { in } e_{2}: \tau} \text { LetForaLL } \\
& \bar{c}=f t v\left(\sigma^{\prime}\right) \backslash \operatorname{dom}(\Gamma) \quad \Gamma^{\prime}=\Gamma, \overline{c: *}, \overline{c \sim \tau} \\
& \overline{\text { isInternalTypeVar }(\tau)} \\
& \frac{\Gamma^{\prime} \Vdash \sigma \leq \sigma^{\prime} \quad \Gamma^{\prime} r_{\mathrm{p}} p: \sigma^{\prime} \Rightarrow \Gamma^{\prime \prime}}{\Gamma \vdash_{\mathrm{p}}\left(p:: \sigma^{\prime}\right): \sigma \Rightarrow \Gamma^{\prime \prime}} \text { PATSigTv }
\end{aligned}
$$

Figure 6. Typing with scoped type variables

GHC has already adopted it, in response to a request ${ }^{9}$ from one of this paper's authors. Thus, despite the addition of equalities in Rule PATCON, we do not have a negative effect on type inference.

\subsection{Closed Pattern Signatures}

Our next step is to formalize PatternSignatures, which allows the user to annotate patterns with type signatures, but for now we will only handle closed pattern signatures. We simply add one new typing rule PATSIG, shown in Fig. 5. Note that the user is allowed to give the pattern a more specific type, as in this example, which requires RankNTypes:

$f::(\forall a . a \rightarrow a) \rightarrow \ln t$

$f(x:: \ln t \rightarrow \ln t)=x 42$

The typing rule expresses this through the premise $\Gamma \Vdash \sigma \leq$ $\sigma^{\prime}$, an appeal to the subtype relation on polytypes. This subtype relationship checks that the expected type of the pattern $\sigma$ is more general than the annotated type $\sigma^{\prime}$. Note that this relationship is backwards from the usual expected/actual relationship in typing because patterns are in a negative position. The subtleties of polytype subtyping are well explored in the literature ${ }^{10}$ and need not derail our exploration here. However, note that Rule PATSIG checks pattern $p$ with the annotated type $\sigma^{\prime}$, not the more general $\sigma$-after all, the user has asked us to use $\sigma^{\prime}$.

\subsection{Scoped Type Variables}

Next, we add support for two features that can bring type variables into scope: open pattern signatures and let with an explicit $\forall$ The grammar now allows a polytype $\sigma$ as the

\footnotetext{
${ }^{9}$ https://ghc.haskell.org/trac/ghc/ticket/15009

${ }^{10} \mathrm{GHC}$ 's current implementation of subtyping is described by Eisenberg et al. [2016], who also cite other relevant publications on the subject.
} 
annotation to a let-bound identifier. We additionally replace PatSig with PAtSigTv and add LetForall in Fig. 6.

The LeTFORALL rule allows programmers to bring variables $\bar{c}$ into scope when an explicit $\forall$ is mentioned in the source. Note that this rule does not do any implicit lexical generalization: echoing GHC's behavior, if the user writes a $\forall$, all new variables to be used in the type signature must be bound explicitly.

In Rule PATSigTv, the last two premises are identical to those of its predecessor PATSig. The first premise extracts the type variables $\bar{c}$ that are free in the user-written type signature $\sigma^{\prime}$, but not already in scope in $\Gamma$. The "not already in scope" part reflects the discussion of Section 3.1.

But what if $\Gamma$ contains a binding, introduced by rule PATCon, for a type variable that just happens to have the same name as one of the $\bar{c}$ in a user-written signature? After all, the names of the type variables in PATCON are arbitrary internal names; they just need to be fresh. Our solution is simple: we take advantage of the difference between internal type variables and external ones. The user cannot accidentally capture an internal variable.

The second (top-right) premise of Rule PATSigTv is the most unusual. It brings the variables $\bar{c}$ into scope, but then also assumes that each variable $c$ equals some other type $\tau$; the following premise asserts that each $\tau$ is, in fact, just an internal type variable $b$. Strikingly, the $\bar{\tau}$ are mentioned nowhere else in the rule. This setup essentially says that the $\bar{c}$ are merely a renaming of existing in-scope internal variables. In practice, the $\bar{\tau}$ are chosen in order to make the subtyping relationship $\Gamma^{\prime} \Vdash \sigma \leq \sigma^{\prime}$ hold; GHC checks this subtyping relationship, unifying the $\bar{c}$ with internal variables $\bar{b}$ as necessary. Because the subtyping relationship is checked with respect to a context that contains the $\overline{c \sim \tau}$ equalities, the $\bar{\tau}$ do not need to be explicitly mentioned again in the rule. For example, consider

$$
\begin{aligned}
& \text { data ExIntegral where } \quad-\text { packs an Integral value } \\
& \qquad M k E x:: \forall a \text {. Integral } a \Rightarrow a \rightarrow \text { ExIntegral } \\
& \text { getInt :: ExIntegral } \rightarrow \text { Integer } \\
& \text { getInt }(M k E x(x:: c))=\text { tolnteger @c } x
\end{aligned}
$$

The pattern match on MkEx brings an internal existential variable $a$ into scope, via the PATCON rule. Recall that the user cannot type the name of such a variable. Instead, the user annotates the pattern $x$ with the user-written type variable c. This annotation triggers Rule PATSigTv, which must find a type $\tau$ such that $a: *, c: *, c \sim \tau \Vdash a \leq c$. The answer is that we must choose $\tau$ to be equal to the variable $a$, and the rule succeeds. We have thus renamed the internal variable $a$ to become the user-written variable $c$ and can successfully use $c$ in the pattern's right-hand side.

Contrast that behavior with this (failing) example:

notAVar :: Int $\rightarrow$ Int

$\operatorname{not} A \operatorname{Var}(x:: c)=x$

$$
\begin{aligned}
& \text { Patterns } \quad p::=x|\mathrm{~K} \overline{@ \tau} \bar{p}| p:: \sigma \\
& \left(\mathrm{K}: \forall{\overline{a_{j}}}^{j} \cdot Q \Rightarrow{\overline{\sigma_{k}}}^{k} \rightarrow \mathrm{T}{\overline{v_{i}}}^{i}\right) \in \Gamma \\
& \bar{c}_{l}^{l}=\operatorname{ftv}\left(\overline{\tau_{j}^{\prime}}{ }^{j}\right) \backslash \operatorname{dom}(\Gamma) \quad{\overline{a_{j}}}^{j} \# \operatorname{dom}(\Gamma) \\
& \Gamma^{\prime}=\Gamma,{\overline{a_{j}: *^{*}}}^{j},{\overline{c_{l}}: *}^{l},{\overline{c_{l} \sim \tau_{l}^{\prime \prime}}}^{l},{\overline{v_{i} \sim \tau_{i}}}^{i}, Q \\
& {\overline{\Gamma^{\prime} \Vdash \tau_{j}^{\prime} \sim a_{j}}}^{j} \quad{\overline{\text { isInternalTypeVar }\left(\tau_{l}^{\prime \prime}\right)}}^{l} \\
& \Gamma^{\prime} \vdash_{\mathrm{p}}^{*}{\overline{p_{k}: \sigma_{k}}}^{k} \Rightarrow \Gamma^{\prime \prime} \\
& \Gamma \vdash_{\mathrm{p}} \mathrm{K}{\overline{@ \tau_{j}^{\prime}}}^{j}{\overline{p_{k}}}^{k}: \mathrm{T}{\overline{\tau_{i}}}^{i} \Rightarrow \Gamma^{\prime \prime}
\end{aligned}
$$

Figure 7. Typing of type applications in patterns

Here, we are trying to bind a user-written type variable $c$ to Int. GHC rejects this function, saying that $c$ does not match with Int. In terms of Rule SigPATTV, there exists no $\tau$ such that $c: *, c \sim \tau \Vdash I n t \leq c$ and isInternalTypeVar $(\tau)$ holds.

There is a free design choice embodied in Rule SigPATTv: the rule asserts that each $c$ must be a renaming of a type variable. Instead, we could simply drop the isInternalTypeVar $(\tau)$, allowing each type variable to rename a type. Nothing else in the system would have to change. Indeed, understanding this very fact is one of the primary motivators for writing this specification in the first place.

\subsection{Type Applications in Patterns}

Having nailed down the status quo, it is now easy to specify what it should mean to use type applications in patterns. This version supports type applications only in constructor patterns; we study pattern synonyms in the appendix of the extended version ${ }^{11}$. The syntax and new typing rule are shown in Fig. 7. Rule PATCONTyApp looks scary, but it just integrates the concepts seen in Rule PATSigTv into Rule PAtCon. We have kept all the iteration indices to help the reader match up which lists are expected to have the same size.

Let us look at each premise separately:

- Once again, the type variables $\bar{c}_{l}^{l}$ are those that occur in the explicit type patterns but are not yet in scope. These are treated like type variables in a pattern signature: they are brought into scope here, each as a short-hand for some type $\tau_{l}^{\prime \prime}$, as long as that type is an internal type variable.

- The environment $\Gamma$ is extended to $\Gamma^{\prime}$ and contains now the (internal) type variables ${\overline{a_{j}}}^{j}$, the user-written scoped type variables $\bar{c}_{l}^{l}$, the type equations equating each $c_{l}$ to its internal type variable $b_{l}$, the GADT equalities ${\overline{v_{i} \sim \tau_{i}}}^{i}$, and the constraint $Q$ captured by $\mathrm{K}$.

- The type patterns are checked against the types they match against. In contrast to pattern signatures, we use type equality here $(\sim)$, not the subtyping relation

\footnotetext{
${ }^{11}$ https://arxiv.org/abs/1806.03476
} 
$(\leq)$ : no types involved can be polytypes, and so the subtyping relation degenerates to type equality.

As written here, the rule requires a type application for each type variable (note that the ${\overline{@ \tau_{j}^{\prime}}}^{j}$ use the same indices as the quantified type variables $\bar{a}_{j}^{j}$ in K's type). However, we can weaken this requirement simply by dropping some $\tau_{j}^{\prime}$ s from both the conclusion and the relevant premises.

Just as in Rule SigPatTv:

- The ${\overline{\tau_{l}^{\prime \prime}}}^{l}$ are mentioned nowhere else in the rule; instead, they are fixed such that the equality constraints for the ${\overline{\tau_{j}^{\prime}}}^{j}$ are entailed by $\Gamma^{\prime}$.

- The rule requires that each user-written type variable stands for an internal variable, but we can once again simply drop the isInternalTypeVar $\left(\tau_{l}^{\prime \prime}\right)$ premise to relax this restriction.

\subsection{Type Safety}

At this point, after developing a set of inference rules defining a type system, one would normally prove that the language is type safe. We do not do so here. Not only would defining an operational semantics and writing out a proof distract us from our main point (the precise description and specification of the use of type variables in patterns), but it is also largely unnecessary. Let us assume that GHC/Haskell, without our new extension, is type safe. (See, e.g., Sulzmann et al. [2007] for a related proof.) If we compare PATCon to our new PATConTyApr, we see that the difference is only the new type variables brought into scope. Yet this same rule insists that these type variables are equal to existing types. In other words, the type variables are merely abbreviations or renamings of other types. Furthermore, the changes have no effect on operational behavior: the changes are all at compile-time. There appears to be no way that introducing such variables can cause a type system to lose safety-everything we have done here amounts only to syntactic convenience ${ }^{12}$, thus obviating the need for a full-blown proof.

\subsection{Conclusion}

Through the incremental building of rules, we can see precisely how the new feature of explicit binding sites for type variables fits into the existing typing framework. We have also explored two further extensions ${ }^{13}$ :

- Allowing type application in patterns headed by pattern synonym [Pickering et al. 2016]. Our framework extends well in this new context, offering no surprises.

- Incorporating explicit binding sites for type variables in the patterns of a $\lambda$-expression. This is slightly subtler

\footnotetext{
${ }^{12}$ In the case that a variable is ambiguous, such as the example in Section 3.6, our new features indeed change what is possible. However, this should be seen more as an infelicity of the way the previous binding structure worked than a new feature we are introducing.

${ }^{13}$ In the appendix of the extended version at https://arxiv.org/abs/1806.03476
}

(though the end result adds only one, simple typing rule), but is relegated to the appendix because it requires reasoning about bidirectional type checking. Bringing all the necessary context into scope would take us too far afield here.

\section{Alternative Approaches}

\subsection{Universals vs. Existentials}

Type theorists are wont to separate quantified type variables in data constructors into two camps: universals and existentials. Here is a contrived but simple example:

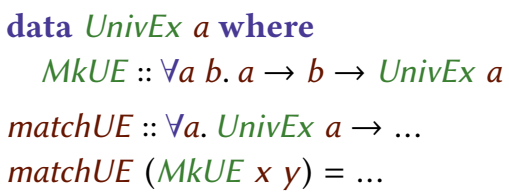

In the constructor $M K U E$, the variable $a$ is universal (it is fixed by the return type UnivEx $a$ ) while $b$ is existential (it is not fixed by the result type). When we match on MkUE in matchUE, we might want to bind $b$, as it is first brought into scope by the match. However, we never need to match $a$, as it is already in scope from matchUE's type signature.

An alternative design for type applications in patterns is to allow matching only existentials in pattern matches, thus:

matchUE :: $\forall a$. UnivEx $a \rightarrow \ldots$.

matchUE (MkUE @bx y) =...

Indeed, this forms the main payload of the original GHC proposal for binding type variables [Suarez 2017]. This design is attractive because the bindings would be concise: only those variables that need to be bound would be available. However, there are two distinct drawbacks:

Universals and existentials are hard to differentiate. Given the complexity of Haskell, the line between these two is blurry. Clearly, $a$ is universal in the constructor $M k U E$ above. But what if its type were MkUE :: $\forall a$ b. $a \rightarrow b \rightarrow$ UnivEx $(I d a)$, where $I d$ is a type synonym? An injective type family? If we add $a \sim b$ to the constraints of MkUE, then $b$ is also fixed by the result type-does that make it a universal?

The question of whether the value of a type variable is fixed by the return type depends on how smart the compiler is, and any specification would have to draw an arbitrary line. In the end, this would leave our users just very confused.

Universals can be instantiated in expressions. When using a data constructor in an expression, the caller is free to instantiate both universals and existentials. Indeed, universals and existentials are utterly indistinguishable in expressions. That means that one might write MkUE @Int @Bool 5 True in an expression. If we could match against only existentials in patterns, though, we would write a pattern MkUE @bxy, remembering to skip the universal $a$. This would both be confusing to users and weaken the ergonomics of patterns, 
whose chief virtue is that deconstructing a datatype resembles closely the syntax of constructing one.

We thus prefer not to differentiate universals and existentials in this way.

\subsection{The Type-Lambda Approach}

A plausible alternative approach to adding scoped type variables is to take a hint from System F, the explicitly-typed polymorphic lambda calculus [Girard 1990]. In System F, a type lambda, written " $\Lambda$ ", binds a type variable, just as a term lambda, written " $\lambda$ ”, binds a term variable. For example:

$$
\begin{aligned}
& i d: \forall \alpha \cdot \alpha \rightarrow \alpha \\
& i d=\Lambda \alpha \cdot \lambda x: \alpha \cdot x
\end{aligned}
$$

A term $\Lambda \alpha . e$ has type $\forall \alpha . \tau$, for some type $\tau$, just as a term $\lambda x . e$ has type $\tau_{1} \rightarrow \tau_{2}$. Hence, a very natural idea is to bind a source-language type variable with a source-language type lambda. This "the type-lambda approach" is the one adopted by SML 97 [Milner et al. 1997]. In SML one can write:

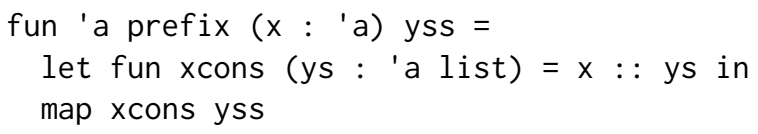

Here, " a" following the keyword fun is the binding site of an (optional) type parameter of prefix; it scopes over the patterns of the definition and its right hand side.

Just as Haskell has implicit quantification in type signatures, SML allows the programmer to introduce implicit type lambdas. This definition is elaborated into the previous one:

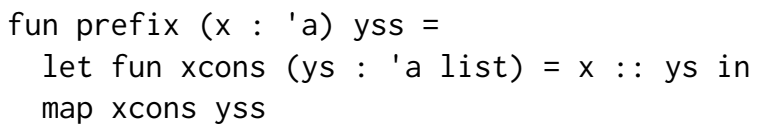

The language definition gives somewhat intricate rules to explain how to place the implicit lambdas. For example:

fun $f x=\ldots$ (fun $\left(y:{ }^{\prime} a\right) \Rightarrow y$ ) ...

Where is the type lambda that binds the type variable ' $a$ ? In SML one cannot answer that question without knowing both what the "...." is, and the context for the definition fun $f$. Roughly speaking, the type lambda for an implicitly-scoped type variable ' $a$ is placed on the innermost function definition that encloses all the free occurrences of ' $a$. The rule [Milner et al. 1997] is only one informal, albeit carefully worded, paragraph; the formal typing rules assume that a pre-processing pass has inserted an explicit binding for every type variable that is implicitly bound by the above rule.

The type-lambda approach explicitly connects lexical scoping and quantification. In contrast, our approach presented here decouples the two, by treating a lexically scoped type variable merely as an alias for a type (or type variable). The appendix, included in the extended version of this paper, additionally has the details of an extension of this work to include explicit binding of type variables in $\lambda$-expressions, providing a similar experience to what we see in SML above.

\section{Acknowledgments}

This material is based upon work supported by the National Science Foundation under Grant No. 1319880, Grant No. 1521539, and Grant No. 1704041.

\section{References}

Joachim Breitner. 2018. Allow ScopedTypeVariables to refer to types. GHC proposal. https://github.com/ghc-proposals/ghc-proposals/pull/128

Manuel M. T. Chakravarty, Gabriele Keller, Simon L. Peyton Jones, and Simon Marlow. 2005. Associated types with class. In POPL. ACM, 1-13. https://doi.org/10.1145/1040305.1040306

Richard A. Eisenberg, Dimitrios Vytiniotis, Simon L. Peyton Jones, and Stephanie Weirich. 2014. Closed type families with overlapping equations. In POPL. ACM, 671-684. https://doi.org/10.1145/2535838.2535856

Richard A. Eisenberg, Stephanie Weirich, and Hamidhasan G. Ahmed. 2016. Visible Type Application. In ESOP (LNCS), Vol. 9632. Springer, 229-254. https://doi.org/10.1007/978-3-662-49498-1_10

Extended version at https://cs.brynmawr.edu/ rae/papers/2016/ type-app/visible-type-app-extended.pdf.

J-Y Girard. 1990. The System F of variable types: fifteen years later. In Logical Foundations of Functional Programming, G Huet (Ed.). Addison-Wesley.

Robin Milner, Mads Tofte, Robert Harper, and David MacQueen. 1997. The Definition of Standard ML (Revised). MIT Press, Cambridge, Massachusetts. xii+114 pages.

Simon L. Peyton Jones, Dimitrios Vytiniotis, Stephanie Weirich, and Mark Shields. 2007. Practical type inference for arbitrary-rank types. fournal of Functional Programming 17, 1 (2007), 1-82. https://doi.org/10.1017/ S0956796806006034

Simon L. Peyton Jones, Stephanie Weirich, Richard A. Eisenberg, and Dimitrios Vytiniotis. 2016. A Reflection on Types. In A List of Successes That Can Change the World - Essays Dedicated to Philip Wadler on the Occasion of His 60th Birthday (LNCS), Vol. 9600. Springer, 292-317. https://doi.org/10.1007/978-3-319-30936-1_16

Matthew Pickering, Gergo Érdi, Simon L. Peyton Jones, and Richard A. Eisenberg. 2016. Pattern synonyms. In Haskell Symposium. ACM, 80-91. https://doi.org/10.1145/2976002.2976013

Tom Schrijvers, Simon L. Peyton Jones, Martin Sulzmann, and Dimitrios Vytiniotis. 2009. Complete and decidable type inference for GADTs. In ICFP. ACM, 341-352. https://doi.org/10.1145/1596550.1596599

Jan Stolarek, Simon L. Peyton Jones, and Richard A. Eisenberg. 2015. Injective type families for Haskell. In Haskell Symposium. ACM, 118-128. https://doi.org/10.1145/2804302.2804314

Emmanuel Suarez. 2017. Binding existential type variables. GHC proposal. https://github.com/ghc-proposals/ghc-proposals/pull/96

Martin Sulzmann, Manuel M. T. Chakravarty, Simon L. Peyton Jones, and Kevin Donnelly. 2007. System F with type equality coercions. In TLDI. ACM, 53-66. https://doi.org/10.1145/1190315.1190324

Dimitrios Vytiniotis, Simon L. Peyton Jones, Tom Schrijvers, and Martin Sulzmann. 2011. OutsideIn(X) Modular type inference with local assumptions. Fournal of Functional Programming 21, 4-5 (2011), 333-412. https://doi.org/10.1017/S0956796811000098 